\title{
ERRATUM
}

\section{Revisiting slope influence in turbulent bedload transport: consequences for vertical flow structure and transport rate scaling - ERRATUM}

doi:10.1017/jfm.2017.903, Published by Cambridge University Press, 25 January 2018

Changes to author affiliations were made incorrectly and the original published article (Maurin, Chauchat \& Frey 2018) has been corrected. The publisher apologises to the authors and readers for this error. The affiliations now appear as follows:

\author{
Raphael Maurin $^{1,} \dagger$, Julien Chauchat ${ }^{2,3}$ and Philippe Frey ${ }^{4}$ \\ ${ }^{1}$ Institut de Mécanique des Fluides de Toulouse (IMFT), Université de Toulouse, CNRS, \\ F-31400 Toulouse, France \\ ${ }^{2}$ CNRS, UMR 5519, LEGI, F-38000 Grenoble, France \\ ${ }^{3}$ Univ. Grenoble Alpes, LEGI, F-38000 Grenoble, France \\ ${ }^{4}$ Univ. Grenoble Alpes, Irstea, UR ETGR, 2 rue de la Papeterie-BP 76, \\ F-38402 St-Martin-d'Hères, France
}

\section{REFERENCE}

Maurin, R., Chauchat, J. \& Frey, P. 2018 Revisiting slope influence in turbulent bedload transport: consequences for vertical flow structure and transport rate scaling. J. Fluid Mech. 839, 135-156. 